\title{
A Difference-Index Based Ranking Bilinear Programming Approach to Solving Bimatrix Games with Payoffs of Trapezoidal Intuitionistic Fuzzy Numbers
}

\author{
Deng-Feng Li and Jie Yang \\ School of Management, Fuzhou University, Fuzhou, Fujian 350108, China \\ Correspondence should be addressed to Deng-Feng Li; dengfengli@sina.com
}

Received 23 October 2013; Accepted 20 November 2013

Academic Editor: Pu-yan Nie

Copyright (c) 2013 D.-F. Li and J. Yang. This is an open access article distributed under the Creative Commons Attribution License, which permits unrestricted use, distribution, and reproduction in any medium, provided the original work is properly cited.

\begin{abstract}
The aim of this paper is to develop a bilinear programming method for solving bimatrix games in which the payoffs are expressed with trapezoidal intuitionistic fuzzy numbers (TrIFNs), which are called TrIFN bimatrix games for short. In this method, we define the value index and ambiguity index for a TrIFN and propose a new order relation of TrIFNs based on the difference index of value index to ambiguity index, which is proven to be a total order relation. Hereby, we introduce the concepts of solutions of TrIFN bimatrix games and parametric bimatrix games. It is proven that any TrIFN bimatrix game has at least one satisfying Nash equilibrium solution, which is equivalent to the Nash equilibrium solution of corresponding parametric bimatrix game. The latter can be obtained through solving the auxiliary parametric bilinear programming model. The method proposed in this paper is demonstrated with a real example of the commerce retailers' strategy choice problem.
\end{abstract}

\section{Introduction}

Bimatrix games are an important type of two-person nonzero-sum noncooperative games, which have been successfully applied to many different areas such as politics, economics, and management. The normal-form bimatrix games assume that the payoffs are represented with crisp values, which indicate that the payoffs are exactly known by players. However, players often are not able to evaluate exactly the payoffs due to imprecision or lack of available information in real game situations. In order to make bimatrix game theory more applicable to real competitive decision problems, the fuzzy set introduced by Zadeh [1] has been used to describe imprecise and uncertain information appearing in bimatrix problems. Using the ranking method of fuzzy numbers, Vidyottama et al. [2] studied the bimatrix game with fuzzy goals and fuzzy payoffs. Using the possibility measure of fuzzy numbers, Maeda [3] introduced two concepts of equilibrium for the bimatrix games with fuzzy payoffs. Bector and Chandra [4] studied bimatrix games with fuzzy payoffs and fuzzy goals based on some duality of fuzzy linear programming. Larbani [5] proposed an approach to solving fuzzy bimatrix games based on the idea of introducing "nature" as a player in fuzzy multiattribute decision-making problems. Atanassov [6] introduced the intuitionistic fuzzy (IF) set (IFS) by adding a nonmembership function, which seems to be suitable for expressing more abundant information. Trapezoidal intuitionistic fuzzy numbers (TrIFNs) are special cases of IFSs defined on the set of real numbers, which can deal with ill-known quantities effectively. And TrIFNs have been playing an important role in fuzzy optimization modeling and decision making [7-10].

This paper will apply the TrIFNs to deal with imprecise quantities in bimatrix game problems, which are called TrIFN bimatrix games for short. Obviously, the TrIFN bimatrix game remarkably differs from fuzzy bimatrix game since the former uses both membership and nonmembership degrees to express the payoffs, while the latter only uses membership degrees to express the payoffs. However, the fuzzy bimatrix game models and methods cannot be directly used to solve TrIFN bimatrix games. Thus, we need to consider the order relation of TrIFNs. Ranking fuzzy numbers are difficult in nature, although there exist a large number of literature on ranking fuzzy numbers [11, 12], IF numbers (IFNs) or IFSs 
[13-18]. Based on the concept of value index and ambiguity index, we propose a difference-index based ranking method of TrIFNs in this paper. This ranking method has good properties such as the linearity. Hereby, a TrIFN bimatrix game is formulated and a solution method is developed on the difference-index based ranking bilinear programming.

The rest of this paper is organized as follows. Section 2 establishes a new order relation of TrIFNs based on the concepts of value index and ambiguity index. Section 3 formulates TrIFN bimatrix games and proposes corresponding solving methodology based on the constructed auxiliary parametric bilinear programming model, which is derived from the new order relation of TrIFNs and the bimatrix game model. In Section 4, the proposed models and method are illustrated with a real example of the commerce retailers' strategy choice problem. Conclusion is drawn in Section 5.

\section{Characteristics and the New Ranking Method of TrIFNs}

2.1. The Definition and Operations of TrIFNs. A TrIFN is expressed as $\widetilde{a}=\left\langle\left(\underline{a}, a_{1}, a_{2}, \bar{a}\right) ; w_{\tilde{a}}, u_{\tilde{a}}\right\rangle$, whose membership function and nonmembership function are defined as follows:

$$
\begin{aligned}
& \mu_{\tilde{a}}(x)= \begin{cases}\frac{(x-\underline{a}) w_{\tilde{a}}}{\left(a_{1}-\underline{a}\right)} & \left(\underline{a} \leq x<a_{1}\right) \\
w_{\tilde{a}} & \left(a_{1} \leq x \leq a_{2}\right) \\
\frac{(\bar{a}-x) w_{\tilde{a}}}{\left(\bar{a}-a_{2}\right)} & \left(a_{2}<x \leq \bar{a}\right) \\
0 & (x<\underline{a}) \text { or }(x>\bar{a}),\end{cases} \\
& v_{\widetilde{a}}(x)= \begin{cases}\frac{\left[a_{1}-x+u_{\tilde{a}}(x-\underline{a})\right]}{\left(a_{1}-\underline{a}\right)} & \left(\underline{a} \leq x<a_{1}\right) \\
u_{\widetilde{a}} & \left(a_{1} \leq x \leq a_{2}\right) \\
\frac{\left[x-a_{2}+u_{\tilde{a}}(\bar{a}-x)\right]}{\left(\bar{a}-a_{2}\right)} & \left(a_{2}<x \leq \bar{a}\right) \\
1 & (x<\underline{a}) \text { or }(x>\bar{a}),\end{cases}
\end{aligned}
$$

respectively, where $w_{\widetilde{A}}$ and $u_{\widetilde{A}}$ represent the maximum membership degree and the minimum nonmembership degree such that they satisfy the following conditions: $0 \leq w_{\widetilde{A}} \leq 1$, $0 \leq u_{\widetilde{A}} \leq 1$, and $0 \leq w_{\widetilde{A}}+u_{\widetilde{A}} \leq 1$.

$\mathrm{A} \operatorname{TrIFN} \tilde{a}=\left\langle\left(\underline{a}, a_{1}, a_{2}, \bar{a}\right) ; w_{\widetilde{a}}, u_{\tilde{a}}\right\rangle$ may express an approximate range of a closed interval $\left[a_{1}, a_{2}\right]$, which is approximately equal to $\left[a_{1}, a_{2}\right]$. Namely, the ill-known quantity "approximate $\left[a_{1}, a_{2}\right]$ " is expressed with any value between $\underline{a}$ and $\bar{a}$ with different membership and nonmembership degrees. It is easily seen that $\mu_{\tilde{a}}(x)+v_{\tilde{a}}(x)=1$ for any $x \in R$ if $w_{\tilde{a}}=1$ and $u_{\tilde{a}}=0$. Hence, the $\operatorname{TrIFN} \tilde{a}=$ $\left\langle\left(\underline{a}, a_{1}, a_{2}, \bar{a}\right) ; w_{\tilde{a}}, u_{\tilde{a}}\right\rangle$ degenerates to $\tilde{a}=\left\langle\left(\underline{a}, a_{1}, a_{2}, \bar{a}\right) ; 1,0\right\rangle$, which is just about a trapezoid fuzzy number. On the other hand, if $a_{1}=a_{2}$, then $\widetilde{a}=\left\langle\left(\underline{a}, a_{1}, a_{2}, \bar{a}\right) ; w_{\tilde{a}}, u_{\tilde{a}}\right\rangle$ degenerates to $\tilde{a}=\left\langle(\underline{a}, a, \bar{a}) ; w_{\tilde{a}}, u_{\tilde{a}}\right\rangle$, which is a TIFN, where $a=a_{1}=a_{2}$.
In a similar way to the arithmetical operations of the trapezoid fuzzy numbers [19], the part arithmetical operations over TrIFNs are stipulated as follows:

$$
\begin{gathered}
\tilde{a}+\tilde{b}=\left\langle\left(\underline{a}+\underline{b}, a_{1}+b_{1}, a_{2}+b_{2}, \bar{a}+\bar{b}\right),\right. \\
\left.w_{\tilde{a}} \wedge w_{\tilde{b}}, u_{\tilde{a}} \vee u_{\tilde{b}}\right\rangle, \\
\gamma \tilde{a}= \begin{cases}\left\langle\left(\gamma \underline{a}, \gamma a_{1}, \gamma a_{2}, \gamma \bar{a}\right) ; w_{\tilde{a}}, u_{\tilde{a}}\right\rangle & (\gamma>0) \\
\left\langle\left(\gamma \bar{a}, \gamma a_{2}, \gamma a_{1}, \gamma \underline{a}\right) ; w_{\tilde{a}}, u_{\tilde{a}}\right\rangle & (\gamma<0),\end{cases}
\end{gathered}
$$

where $\lambda \neq 0$ is a real number and the symbols " $\wedge$ " and " $\vee$ " are the minimum and maximum operators, respectively.

\subsection{Value and Ambiguity of a TrIFN}

Definition 1. A $\alpha$-cut set of a TrIFN $\tilde{a}$ is a crisp subset of $R$, which can be expressed as $\tilde{a}_{\alpha}=\left\{x \mid \mu_{\widetilde{a}}(x) \geq \alpha\right\}$, where $0 \leq$ $\alpha \leq w_{\tilde{a}}$. A $\beta$-cut set of a TrIFN $\tilde{a}$ is a crisp subset of $R$, which can be expressed as $\tilde{a}_{\beta}=\left\{x \mid v_{\widetilde{a}}(x) \leq \beta\right\}$, where $u_{\widetilde{a}} \leq \beta \leq 1$. It directly follows that $\widetilde{a}_{\alpha}$ and $\tilde{a}_{\beta}$ are closed intervals, denoted by $\tilde{a}_{\alpha}=\left[L_{\widetilde{a}}(\alpha), R_{\widetilde{a}}(\alpha)\right]$ and $\tilde{a}_{\beta}=\left[L_{\widetilde{a}}(\beta), R_{\widetilde{a}}(\beta)\right]$, respectively, which can be calculated as follows:

$$
\begin{gathered}
\tilde{a}_{\alpha}=\left[\frac{\left[\left(w_{\tilde{a}}-\alpha\right) \underline{a}+\alpha a_{1}\right]}{w_{\tilde{a}}}, \frac{\left[\left(w_{\tilde{a}}-\alpha\right) \bar{a}+\alpha a_{2}\right]}{w_{\tilde{a}}}\right], \\
\tilde{a}_{\beta}=\left[\frac{\left[(1-\beta) a_{1}+\left(\beta-u_{\tilde{a}}\right) \underline{a}\right]}{\left(1-u_{\tilde{a}}\right)}, \frac{\left[(1-\beta) a_{2}+\left(\beta-u_{\tilde{a}}\right) \bar{a}\right]}{\left(1-u_{\tilde{a}}\right)}\right] .
\end{gathered}
$$

Definition 2. Let $\tilde{a}_{\alpha}$ and $\tilde{a}_{\beta}$ be a $\alpha$-cut number and $\beta$-cut number of a TrIFN $\tilde{a}$, respectively. Then the values of the membership and nonmembership functions for the TrIFN $\tilde{a}$ are defined as follows:

$$
\begin{aligned}
& V_{\mu}(\widetilde{a})=\int_{0}^{w_{\widetilde{a}}}\left[\frac{L_{\widetilde{a}}(\alpha)+R_{\widetilde{a}}(\alpha)}{2}\right] f(\alpha) \mathrm{d} \alpha, \\
& V_{v}(\widetilde{a})=\int_{u_{\tilde{a}}}^{1}\left[\frac{L_{\widetilde{a}}(\beta)+R_{\widetilde{a}}(\beta)}{2}\right] g(\beta) \mathrm{d} \beta,
\end{aligned}
$$

where $f(\alpha)$ is a nonnegative and nondecreasing function on the interval $\left[0, w_{\tilde{a}}\right]$ with $f(0)=0$ and $f\left(w_{\tilde{a}}\right)=1$ and $g(\beta)$ is a nonnegative and nonincreasing function on the interval $\left[u_{\tilde{a}}, 1\right]$ with $g\left(u_{\tilde{a}}\right)=1$ and $g(1)=0$. Obviously, $f(\alpha)$ and $g(\beta)$ can be considered as weighting functions and have various specific forms in actual applications, which can be chosen according to the real-life situations. In the following, we choose $f(\alpha)=\alpha / w_{\widetilde{a}}\left(\alpha \in\left[0, w_{\widetilde{a}}\right]\right)$ and $g(\beta)=(1-\beta) /\left(1-u_{\widetilde{a}}\right)$ $\left(\beta \in\left[u_{\tilde{a}}, 1\right]\right)$.

The function $f(\alpha)$ gives different weights to elements at different $\alpha$-cuts, so that it can lessen the contribution of the lower $\alpha$-cuts, since these cuts arising from values of $\mu_{\tilde{a}}(x)$ have a considerable amount of uncertainty. Therefore, $V_{\mu}(\widetilde{a})$ and $V_{v}(\widetilde{a})$ synthetically reflect the information on membership and nonmembership degrees. 
According to (5) and (7), we can calculate the value of the membership function as follows:

$$
\begin{aligned}
V_{\mu}(\widetilde{a}) & =\int_{0}^{w_{\tilde{a}}}\left[\frac{\left(w_{\widetilde{a}}-\alpha\right) \underline{a}+\alpha a_{1}}{2 w_{\tilde{a}}}+\frac{\left(w_{\tilde{a}}-\alpha\right) \bar{a}+\alpha a_{2}}{2 w_{\tilde{a}}}\right] \frac{\alpha}{w_{\widetilde{a}}} \mathrm{~d} \alpha \\
& =\frac{\left(\underline{a}+2 a_{1}+2 a_{2}+\bar{a}\right) w_{\widetilde{a}}}{12} .
\end{aligned}
$$

In a similar way, according to (6) and (8), the value of the nonmembership function can be obtained as follows:

$$
V_{v}(\widetilde{a})=\frac{\left(\underline{a}+2 a_{1}+2 a_{2}+\bar{a}\right)\left(1-u_{\tilde{a}}\right)}{12} .
$$

It is directly derived from the condition $0 \leq w_{\tilde{a}}+u_{\tilde{a}} \leq 1$ that $0 \leq V_{\mu}(\widetilde{a}) \leq V_{v}(\widetilde{a})$, which may be concisely expressed as an interval $\left[V_{\mu}(\widetilde{a}), V_{v}(\widetilde{a})\right]$. It is easily proven that the following conclusion holds.

Theorem 3. Assume that $\tilde{a}$ and $\widetilde{b}$ are two TrIFNs with $w_{\tilde{a}}=w_{\tilde{b}}$ and $u_{\widetilde{a}}=u_{\tilde{b}}$. Then, $V_{\mu}(\widetilde{a}+\widetilde{b})=V_{\mu}(\widetilde{a})+V_{\mu}(\widetilde{b})$.

Proof. According to (3) and (9), we can prove that

$$
\begin{aligned}
V_{\mu}(\tilde{a}+\tilde{b})= & \frac{\left(\underline{a}+2 a_{1}+2 a_{2}+\bar{a}\right) w_{\tilde{a}}}{12} \\
& +\frac{\left(\underline{b}+2 b_{1}+2 b_{2}+\bar{b}\right) w_{\tilde{b}}}{12} \\
= & V_{\mu}(\widetilde{a})+V_{\mu}(\widetilde{b}) .
\end{aligned}
$$

In the same way, using (3) and (10), we can prove that $V_{v}(\widetilde{a}+\tilde{b})=V_{v}(\widetilde{a})+V_{v}(\widetilde{b})$.

Definition 4. Let $\tilde{a}_{\alpha}$ and $\tilde{a}_{\beta}$ be a $\alpha$-cut set and $\beta$-cut set of a $\operatorname{TrIFN} \tilde{a}$, respectively. The ambiguities of the membership and nonmembership functions for the TrIFN $\tilde{a}$ are defined as follows:

$$
\begin{aligned}
& A_{\mu}(\widetilde{a})=\int_{0}^{w_{\widetilde{a}}}\left(R_{\tilde{a}}(\alpha)-L_{\widetilde{a}}(\alpha)\right) f(\alpha) \mathrm{d} \alpha, \\
& A_{v}(\widetilde{a})=\int_{\mathcal{U}_{\tilde{a}}}^{1}\left(R_{\widetilde{a}}(\beta)-L_{\widetilde{a}}(\beta)\right) g(\beta) \mathrm{d} \beta .
\end{aligned}
$$

According to (5) and (6), (12) can be obtained as follows:

$$
\begin{gathered}
A_{\mu}(\widetilde{a})=\frac{\left(\bar{a}-\underline{a}+2 a_{2}-2 a_{1}\right) w_{\widetilde{a}}}{6} \\
A_{v}(\widetilde{a})=\frac{\left(\bar{a}-\underline{a}+2 a_{2}-2 a_{1}\right)\left(1-u_{\tilde{a}}\right)}{6} .
\end{gathered}
$$

Obviously, $A_{\mu}(\widetilde{a}) \leq A_{v}(\widetilde{a})$, which can be expressed as an interval $\left[A_{\mu}(\widetilde{a}), A_{v}(\widetilde{a})\right]$.

Theorem 5. Assume that $\tilde{a}$ and $\widetilde{b}$ are two TrIFNs with $w_{\widetilde{a}}=w_{\tilde{b}}$ and $u_{\widetilde{a}}=u_{\tilde{b}}$. Then, $A_{\mu}(\widetilde{a}+\widetilde{b})=A_{\mu}(\widetilde{a})+A_{\mu}(\widetilde{b})$.
Proof. According to (3) and (13), we can prove that

$$
\begin{aligned}
A_{\mu}(\tilde{a}+\widetilde{b})= & \frac{\left(\bar{a}-\underline{a}+2 a_{2}-2 a_{1}\right) w_{\tilde{a}}}{6} \\
& +\frac{\left(\bar{b}-\underline{b}+2 b_{2}-2 b_{1}\right) w_{\tilde{b}}}{6} \\
= & A_{\mu}(\widetilde{a})+A_{\mu}(\tilde{b}) .
\end{aligned}
$$

Similarly, using (3) and (14), we can prove that $A_{v}(\widetilde{a}+\widetilde{b})=$ $A_{v}(\widetilde{a})+A_{v}(\widetilde{b})$.

2.3. The Difference-Index Based Ranking Method. Based on the value and ambiguity of a TrIFN $\widetilde{a}$, its value index and an ambiguity index are defined as follows. Hereby, a new ranking method of TrIFNs is proposed.

Definition 6. Let $\tilde{a}$ be a TrIFN. Its value index and an ambiguity index are defined as follows:

$$
\begin{gathered}
V_{\lambda}(\widetilde{a})=V_{\mu}(\widetilde{a})+\lambda\left(V_{v}(\widetilde{a})-V_{\mu}(\widetilde{a})\right), \\
A_{\lambda}(\widetilde{a})=A_{v}(\widetilde{a})-\lambda\left(A_{v}(\widetilde{a})-A_{\mu}(\widetilde{a})\right),
\end{gathered}
$$

where $\lambda \in[0,1]$ is a weight which represents the decision maker's preference information. $\lambda \in[0,1 / 2)$ shows that decision maker prefers to uncertainty or negative feeling, who is a pessimist; $\lambda \in(1 / 2,1]$ shows that the decision maker prefers to certainty or positive feeling, who is an optimist; $\lambda=1 / 2$ shows that the decision maker is a neutralist, between positive feeling and negative feeling. Therefore, the value-index and the ambiguity-index may reflect the decision maker's subjectivity attitude to the TrIFN.

A difference index of the value index to the ambiguity index for a TrIFN $\tilde{a}$ is defined as follows:

$$
D_{\lambda}(\widetilde{a})=V_{\lambda}(\widetilde{a})-A_{\lambda}(\widetilde{a}) .
$$

Theorem 7. Assume that $\tilde{a}$ and $\tilde{b}$ are two TrIFNs with $w_{\tilde{a}}=$ $w_{\tilde{b}}$ and $u_{\widetilde{a}}=u_{\tilde{b}}$. Then, $D_{\lambda}(\widetilde{a}+\widetilde{b})=D_{\lambda}(\widetilde{a})+D_{\lambda}(\widetilde{b})$, where $\lambda \in[0,1]$.

Proof. According to Theorems 3 and 5, it is derived from (18) that

$$
\begin{aligned}
D_{\lambda}(\widetilde{a}+\widetilde{b}) & =V_{\lambda}(\widetilde{a}+\widetilde{b})-A_{\lambda}(\widetilde{a}+\widetilde{b}) \\
& =\left(V_{\lambda}(\widetilde{a})+V_{\lambda}(\widetilde{b})\right)-\left(A_{\lambda}(\widetilde{a})+A_{\lambda}(\widetilde{b})\right) \\
& =\left(V_{\lambda}(\widetilde{a})-A_{\lambda}(\widetilde{a})\right)+\left(V_{\lambda}(\widetilde{b})-A_{\lambda}(\widetilde{b})\right) \\
& =D_{\lambda}(\widetilde{A})+D_{\lambda}(\widetilde{b}) .
\end{aligned}
$$

Theorem 7 shows that the difference index $D_{\lambda}(\widetilde{a})$ is a linear function of any TrIFN. Furthermore, it can be easily seen that the larger the difference index, the bigger the TrIFN. Thus, we propose the difference index based ranking method of TrIFNs as follows. 
Definition 8. Assume that $\lambda \in[0,1]$. For any TrIFNs $\tilde{a}$ and $\tilde{b}$, we stipulate the following:

(1) $D_{\lambda}(\widetilde{a})>D_{\lambda}(\widetilde{b})$ if and only if $\tilde{a}$ is bigger than $\tilde{b}$, denoted by $\widetilde{a}>\widetilde{b}$;

(2) $D_{\lambda}(\widetilde{a})=D_{\lambda}(\widetilde{b})$ if and only if $\tilde{a}$ is equal to $\tilde{b}$, denoted by $\widetilde{a}=\widetilde{b}$;

(3) $D_{\lambda}(\widetilde{a}) \geq D_{\lambda}(\tilde{b})$ if and only if $\tilde{a}>\tilde{b}$ or $\tilde{a}=\tilde{b}$.

The above ranking method has some useful properties, which satisfy five of the seven axioms proposed by Wang and Kerre [20] that serve as the reasonable properties for the ordering of fuzzy quantities. And the proposed ranking method is two-index, which is used to aggregate both value index and the ambiguity index. Especially, this proposed ranking method has the linearity.

\section{Bilinear Programming Models for TrIFN Bimatrix Games}

3.1. Bimatrix Games and Auxiliary Bilinear Programming Models. Assume that $S_{1}=\left\{\alpha_{1}, \alpha_{2}, \ldots, \alpha_{m}\right\}$ and $S_{2}=$ $\left\{\beta_{1}, \beta_{2}, \ldots, \beta_{n}\right\}$ are sets of pure strategies for players I and II, respectively. The payoff matrices of players I and II are expressed with $\mathbf{A}=\left(a_{i j}\right)_{m \times n}$ and $\mathbf{B}=\left(b_{i j}\right)_{m \times n}$, respectively. The vectors $\mathbf{y}=\left(y_{1}, y_{2}, \ldots, y_{m}\right)^{T}$ and $\mathbf{z}=\left(z_{1}, z_{2}, \ldots, z_{n}\right)^{T}$ are mixed strategies for players I and II, where $y_{i}(i=1,2, \ldots, m)$ and $z_{j}(j=1,2, \ldots, n)$ are probabilities in which players I and II choose their pure strategies $\alpha_{i} \in S_{1}(i=1,2, \ldots, m)$ and $\beta_{j} \in S_{2}(j=1,2, \ldots, n)$, respectively; the symbol " $T$ " is the transpose of a vector/matrix. Sets of all mixed strategies for I and II are denoted by $Y$ and $Z$; that is, $Y=\left\{\mathbf{y} \mid \sum_{i=1}^{m} y_{i}=\right.$ $\left.1, y_{i} \geq 0(i=1,2, \ldots, m)\right\}$, and $Z=\left\{\mathbf{z} \mid \sum_{j=1}^{n} z_{j}=1, z_{j} \geq\right.$ $0(j=1,2, \ldots, n)\}$, respectively. Thus, a two-person nonzerosum finite game may be expressed with $(Y, Z, \mathbf{A}, \mathbf{B})$. In the sequent, such a game usually is simply called the bimatrix game $(\mathbf{A}, \mathbf{B})$ in which both players want to maximize his/her own payoffs. When I chooses any mixed strategy y $\in Y$ and II chooses any mixed strategy $\mathbf{z} \in Z$, the expected payoffs of I and II can be computed as $E_{1}(\mathbf{y}, \mathbf{z})=\mathbf{y}^{T} \mathbf{A z}=$ $\sum_{i=1}^{m} \sum_{j=1}^{n} y_{i} a_{i j} z_{j}$ and $E_{2}(\mathbf{y}, \mathbf{z})=\mathbf{y}^{T} \mathbf{B z}=\sum_{i=1}^{m} \sum_{j=1}^{n} y_{i} b_{i j} z_{j}$, respectively.

Definition 9 (see [21]). If there is a pair $\left(\mathbf{y}^{*}, \mathbf{z}^{*}\right) \in Y \times Z$, such that $\mathbf{y}^{T} \mathbf{A} \mathbf{z}^{*} \leq \mathbf{y}^{* T} \mathbf{A} \mathbf{z}^{*}$ for any $\mathbf{y} \in Y$ and $\mathbf{y}^{* T} \mathbf{B z} \leq \mathbf{y}^{* T} \mathbf{B} \mathbf{z}^{*}$ for any $\mathbf{z} \in Z$, then $\left(\mathbf{y}^{*}, \mathbf{z}^{*}\right)$ is called a Nash equilibrium point of the bimatrix game $(\mathbf{A}, \mathbf{B}), \mathbf{y}^{*}$ and $\mathbf{z}^{*}$ are called Nash equilibrium strategies of players I and II, $u^{*}=\mathbf{y}^{* T} \mathbf{A} \mathbf{z}^{*}$ and $v^{*}=\mathbf{y}^{* T} \mathbf{B z}^{*}$ are called Nash equilibrium values of players I and II, respectively, and $\left(\mathbf{y}^{* T}, \mathbf{z}^{* T}, u^{*}, v^{*}\right)$ is called a Nash equilibrium solution of the bimatrix game $(\mathbf{A}, \mathbf{B})$.

The following theorem guarantees the existence of Nash equilibrium solutions of any bimatrix game.

Theorem 10 (see [22]). Any bimatrix game (A, B) has at least one Nash equilibrium solution.
A Nash equilibrium solution of any bimatrix game (A, B) can be obtained by solving the bilinear programming model stated as in the following Theorem 11.

Theorem 11 (see [23]). Let (A, B) be any bimatrix game. $\left(\mathbf{y}^{* T}, \mathbf{z}^{* T}, u^{*}, v^{*}\right)$ is a Nash equilibrium solution of the bimatrix game (A, B) if and only if it is a solution of the bilinear programming model, which is shown as follows:

$$
\begin{gathered}
\max \left\{\mathbf{y}^{T}(\mathbf{A}+\mathbf{B}) \mathbf{z}-u-v\right\} \\
\text { s.t. }\left\{\begin{array}{c}
\mathbf{A z} \leq u \mathbf{e}^{m} \\
\mathbf{B}^{T} \mathbf{y} \leq v \mathbf{e}^{n} \\
\mathbf{y}^{T} \mathbf{e}^{m}=1 \\
\mathbf{z}^{T} \mathbf{e}^{n}=1 \\
\mathbf{y} \geq 0, \quad \mathbf{z} \geq 0 .
\end{array}\right.
\end{gathered}
$$

Furthermore, if $\left(\mathbf{y}^{* T}, \mathbf{z}^{* T}, u^{*}, v^{*}\right)$ is a solution of the above bilinear programming model, then $u^{*}=\mathbf{y}^{* T} \mathbf{A z}^{*}, v^{*}=$ $\mathbf{y}^{* T} \mathbf{B} \mathbf{z}^{*}$, and $\mathbf{y}^{* T}(\mathbf{A}+\mathbf{B}) \mathbf{z}^{*}-u^{*}-v^{*}=0$.

3.2. Models and Method for TrIFN Bimatrix Games. Let us consider a TrIFN bimatrix game, where sets of pure strategies $S_{1}$ and $S_{2}$ and sets of mixed strategies $Y$ and $Z$ for players I and II are defined as in the above sections. If player I chooses any pure strategy $\alpha_{i} \in S_{1}(i=1,2, \ldots, m)$ and player II chooses any pure strategy $\beta_{j} \in S_{2}(j=1,2, \ldots, n)$, then at the situation $\left(\alpha_{i}, \beta_{j}\right)$ players I and II gain payoffs, which are expressed with TrIFNs $\widetilde{A}_{i j}\left(\alpha_{i}, \beta_{j}\right)=\left\{\left\langle\left(\alpha_{i}, \beta_{j}\right) ;\left(\underline{a}_{i j}, a_{1 i j}, a_{2 i j}, \bar{a}_{i j}\right), w_{\widetilde{A}_{i j}}, u_{\widetilde{A}_{i j}}\right\rangle\right\}$ and $\widetilde{B}_{i j}\left(\alpha_{i}, \beta_{j}\right)=\left\{\left\langle\left(\alpha_{i}, \beta_{j}\right) ;\left(\underline{b}_{i j}, b_{1 i j}, b_{2 i j}, \bar{b}_{i j}\right), w_{\widetilde{A}_{i j}}, u_{\widetilde{A}_{i j}}\right\rangle\right\}(i=1$, $2, \ldots, m ; j=1,2, \ldots, n)$, respectively. Thus, the payoff matrices of players I and II are expressed as $\widetilde{\mathbf{A}}=\left(\widetilde{A}_{i j}\left(\alpha_{i}, \beta_{j}\right)\right)_{m \times n}$ and $\widetilde{\mathbf{B}}=\left(\widetilde{B}_{i j}\left(\alpha_{i}, \beta_{j}\right)\right)_{m \times n}$, respectively.

As stated earlier, the above payoffs $\widetilde{A}_{i j}\left(\alpha_{i}, \beta_{j}\right)$ and $\widetilde{B}_{i j}\left(\alpha_{i}, \beta_{j}\right)(i=1,2, \ldots, m ; j=1,2, \ldots, n)$ of players I and II are TrIFNs in the finite universal set $X^{\prime}=\left\{\left(\alpha_{i}, \beta_{j}\right)\right.$ | $\left.\alpha_{i} \in S_{1}(i=1,2, \ldots, m), \beta_{j} \in S_{2}(j=1,2, \ldots, n)\right\}$. For instance, let us consider a simple example in which there are two pure strategies for both players I and II; that is, player I has pure strategies $\alpha_{1}$ and $\alpha_{2}$ and player II has pure strategies $\beta_{1}$ and $\beta_{2}$. Then, the universal set is $X_{0}^{\prime}=\left\{\left(\alpha_{1}, \beta_{1}\right),\left(\alpha_{1}, \beta_{2}\right),\left(\alpha_{2}, \beta_{1}\right),\left(\alpha_{2}, \beta_{2}\right)\right\}$, which has four elements (i.e., situations). In the sequel, the above TrIFN bimatrix game is simply denoted by $(\widetilde{\mathbf{A}}, \widetilde{\mathbf{B}})$ for short. If player I chooses any mixed strategy y $\in Y$ and player II chooses any mixed strategy $\mathbf{z} \in Z$, then the expected payoff of player I is $\widetilde{E}_{1}(\mathbf{y}, \mathbf{z})=\mathbf{y}^{T} \widetilde{\mathbf{A}} \mathbf{z}$. According to the operations of TrIFNs, the expected payoff $\widetilde{E}_{1}(\mathbf{y}, \mathbf{z})$ of player I is a TrIFN and can be calculated: $\widetilde{E}_{1}(\mathbf{y}, \mathbf{z})=\left\{\left\langle(\bar{y}, \bar{z}), \sum_{i=1}^{n} \sum_{j=1}^{n} y_{i}\left(\underline{a}_{i j}, a_{1 i j}, a_{2 i j}, \bar{a}_{i j}\right) z_{j} ;\right.\right.$ $\left.\min \left\{w_{\widetilde{a}_{i j}}\right\}, \max \left\{u_{\tilde{a}_{i j}}\right\}\right\rangle$, where $(\bar{y}, \bar{z})$ represents a mixed situation, which corresponds to the mixed strategies $\mathbf{y}$ and $\mathbf{z}$.

Similarly, the expected payoff of player II is $\widetilde{E}_{2}(\mathbf{y}, \mathbf{z})=\mathbf{y}^{T} \widetilde{\mathbf{B}} \mathbf{z}$, which can be calculated: $\widetilde{E}_{2}(\mathbf{y}, \mathbf{z})=$ $\left.\left.\sum_{i=1}^{n} \sum_{j=1}^{n} y_{i}\left(\underline{b}_{i j}, b_{1 i j}, b_{2 i j}, \bar{b}_{i j}\right) z_{j} ; \min \left\{w_{\widetilde{a}_{i j}}\right\}, \max \left\{u_{\widetilde{a}_{i j}}\right\}\right\rangle\right\}$, where 
$\left(\bar{y}^{\prime}, \bar{z}^{\prime}\right)$ represents a mixed situation, which corresponds to the mixed strategies $\mathbf{y}$ and $\mathbf{z}$.

Definition 12. Assume that there is a pair $\left(\mathbf{y}^{*}, \mathbf{z}^{*}\right) \in Y \times Z$. If any $\mathbf{y} \in Y$ and $\mathbf{z} \in Z$ satisfy $\mathbf{y}^{T} \widetilde{\mathbf{A}} \mathbf{z}^{*} \leq_{\operatorname{TrIFN}} \mathbf{y}^{* T} \widetilde{\mathbf{A}} \mathbf{z}^{*}$ and $\mathbf{y}^{* T} \widetilde{\mathbf{B}} \mathbf{z} \leq_{\text {TrIFN }} \mathbf{y}^{* T} \widetilde{\mathbf{B}} \mathbf{z}^{*}$, then $\left(\mathbf{y}^{*}, \mathbf{z}^{*}\right)$ is called a Nash equilibrium point of the $\operatorname{TrIFN}$ bimatrix game $(\widetilde{\mathbf{A}}, \widetilde{\mathbf{B}}), \mathbf{y}^{*}$ and $\mathbf{z}^{*}$ are called Nash equilibrium strategies of players I and II, $\widetilde{u}^{*}=$ $\mathbf{y}^{* T} \widetilde{\mathbf{A}} \mathbf{z}^{*}$ and $\widetilde{v}^{*}=\mathbf{y}^{* T} \widetilde{\mathbf{B}} \mathbf{z}^{*}$ are called Nash equilibrium values of players I and II, respectively, and $\left(\mathbf{y}^{*}, \mathbf{z}^{*}, \widetilde{u}^{*}, \widetilde{v}^{*}\right)$ is called a Nash equilibrium solution of the TrIFN bimatrix game $(\widetilde{\mathbf{A}}, \widetilde{\mathbf{B}})$.

Stated as earlier, however, player I's expected payoff $\mathbf{y}^{T} \widetilde{\mathbf{A}} \mathbf{z}$ and player II's expected payoff $\mathbf{y}^{T} \widetilde{\mathbf{B}} \mathbf{z}$ are TrIFNs. Therefore, there are no commonly used concepts of solutions of TrIFN bimatrix games. Furthermore, it is not easy to compute the membership degrees and the nonmembership degrees of players' expected payoffs. As a result, solving Nash equilibrium solutions of TrIFN bimatrix games is very difficult. In the sequel, we use the ranking function $D_{\lambda}$ to develop a new method for solving the TrIFN bimatrix game $(\widetilde{\mathbf{A}}, \widetilde{\mathbf{B}})$.

Using the ranking function of TrIFNs given by (18), we can transform the TrIFN payoff matrices $\widetilde{\mathbf{A}}$ and $\widetilde{\mathbf{B}}$ of players I and II into the payoff matrices as follows:

$$
\begin{aligned}
& \widetilde{\mathbf{A}}_{\lambda_{1}}=D_{\lambda_{1}}\left(\left(\widetilde{\mathbf{A}}_{i j}\left(\alpha_{i}, \beta_{j}\right)\right)_{m \times n}\right)=\left(D_{\lambda_{1}}\left(\widetilde{\mathbf{A}}_{i j}\left(\alpha_{i}, \beta_{j}\right)\right)\right)_{m \times n}, \\
& \widetilde{\mathbf{B}}_{\lambda_{2}}=D_{\lambda_{2}}\left(\left(\widetilde{\mathbf{B}}_{i j}\left(\alpha_{i}, \beta_{j}\right)\right)_{m \times n}\right)=\left(D_{\lambda_{2}}\left(\widetilde{\mathbf{B}}_{i j}\left(\alpha_{i}, \beta_{j}\right)\right)\right)_{m \times n},
\end{aligned}
$$

where $\lambda_{1} \in[0,1], \lambda_{2} \in[0,1], D_{\lambda_{1}}\left(\widetilde{\mathbf{A}}_{i j}\left(\alpha_{i}, \beta_{j}\right)\right)=V_{\widetilde{\mathbf{A}}_{i j}\left(\alpha_{i}, \beta_{j}\right)}-$ $A_{\widetilde{\mathbf{A}}_{i j}\left(\alpha_{i}, \beta_{j}\right)}$, and $D_{\lambda_{2}}\left(\widetilde{\mathbf{B}}_{i j}\left(\alpha_{i}, \beta_{j}\right)\right)=V_{\widetilde{\mathbf{B}}_{i j}\left(\alpha_{i}, \beta_{j}\right)}-A_{\widetilde{\mathbf{B}}_{i j}\left(\alpha_{i}, \beta_{j}\right)}(i=$ $1,2, \ldots, m ; j=1,2, \ldots, n)$.

According to the above usage and notations, the above parametric bimatrix game can be simply denoted by $\left(\widetilde{\mathbf{A}}_{\lambda_{1}}, \widetilde{\mathbf{B}}_{\lambda_{2}}\right)$, where the pure (or mixed) strategy sets of players I and II are $S_{1}$ and $S_{2}$ (or $Y$ and $Z$ ) defined as mentioned above. Then, the TrIFN bimatrix game $(\widetilde{\mathbf{A}}, \widetilde{\mathbf{B}})$ is transformed into the parametric bimatrix game $\left(\widetilde{\mathbf{A}}_{\lambda_{1}}, \widetilde{\mathbf{B}}_{\lambda_{2}}\right)$. Hereby, according to Definitions 8-12 and Theorem 7, we can give the definition of satisfying Nash equilibrium solutions of the TrIFN bimatrix game $\left(\widetilde{\mathbf{A}}_{\lambda_{1}}, \widetilde{\mathbf{B}}_{\lambda_{2}}\right)$ as follows.

Definition 13. For given parameters $\lambda_{1} \in[0,1]$ and $\lambda_{2} \epsilon$ $[0,1]$, if there is a pair $\left(\mathbf{y}^{*}, \mathbf{z}^{*}\right) \in Y \times Z$, such that any $\mathbf{y} \in Y$ and $\mathbf{z} \in Z$ satisfy the following conditions: $\mathbf{y}^{T} \widetilde{\mathbf{A}}_{\lambda_{1}} \mathbf{z}^{*} \leq$ $\mathbf{y}^{* T} \widetilde{\mathbf{A}}_{\lambda_{1}} \mathbf{z}^{*}$ and $\mathbf{y}^{* T} \widetilde{\mathbf{B}}_{\lambda_{2}} \mathbf{z} \leq \mathbf{y}^{* T} \widetilde{\mathbf{B}}_{\lambda_{2}} \mathbf{z}^{*}$, then $\left(\mathbf{y}^{*}, \mathbf{z}^{*}\right)$ is called a satisfying Nash equilibrium point of the TrIFN bimatrix game $\left(\widetilde{\mathbf{A}}_{\lambda_{1}}, \widetilde{\mathbf{B}}_{\lambda_{2}}\right), \mathbf{y}^{*}$ and $\mathbf{z}^{*}$ are called satisfying Nash equilibrium strategies of players I and II, $u^{*}\left(\lambda_{1}\right)=\mathbf{y}^{* T} \widetilde{\mathbf{A}}_{\lambda_{1}} \mathbf{z}^{*}$ and $v^{*}\left(\lambda_{2}\right)=$ $\mathbf{y}^{* T} \widetilde{\mathbf{B}}_{\lambda_{2}} \mathbf{z}^{*}$ are called satisfying equilibrium values of players I and II, respectively, and $\left(\mathbf{y}^{*}, \mathbf{z}^{*}, u^{*}\left(\lambda_{1}\right), v^{*}\left(\lambda_{2}\right)\right)$ is called a satisfying Nash equilibrium solution of the TrIFN bimatrix game $\left(\widetilde{\mathbf{A}}_{\lambda_{1}}, \widetilde{\mathbf{B}}_{\lambda_{2}}\right)$.
It can be easily seen from the ranking function given by (18) and Theorem 7 that Definitions 12 and 13 are equivalent in the sense of the order relation defined by Definition 8 . Thus, for given parameters $\lambda_{1} \in[0,1]$ and $\lambda_{2} \in[0,1]$, according to Theorem 10, the parametric bimatrix game $\left(\widetilde{\mathbf{A}}_{\lambda_{1}}, \widetilde{\mathbf{B}}_{\lambda_{2}}\right)$ has at least one Nash equilibrium solution. Namely, the TrIFN bimatrix game $\left(\widetilde{\mathbf{A}}_{\lambda_{1}}, \widetilde{\mathbf{B}}_{\lambda_{2}}\right)$ has at least one satisfying Nash equilibrium solution, which can be obtained through solving the following parametric bilinear programming model according to Theorem 11:

$$
\begin{aligned}
& \max \left\{\sum _ { j = 1 } ^ { n } \sum _ { i = 1 } ^ { m } y _ { i } \left[V_{\lambda 1}\left(\widetilde{A}_{i j}\left(\alpha_{i}, \beta_{j}\right)\right)-A_{\lambda 1}\left(\widetilde{A}_{i j}\left(\alpha_{i}, \beta_{j}\right)\right)\right.\right. \\
& \left.+V_{\lambda 2}\left(\widetilde{B}_{i j}\left(\alpha_{i}, \beta_{j}\right)\right)-A_{\lambda 2}\left(\widetilde{B}_{i j}\left(\alpha_{i}, \beta_{j}\right)\right)\right] z_{j} \\
& \left.-u\left(\lambda_{1}\right)-v\left(\lambda_{2}\right)\right\}
\end{aligned}
$$

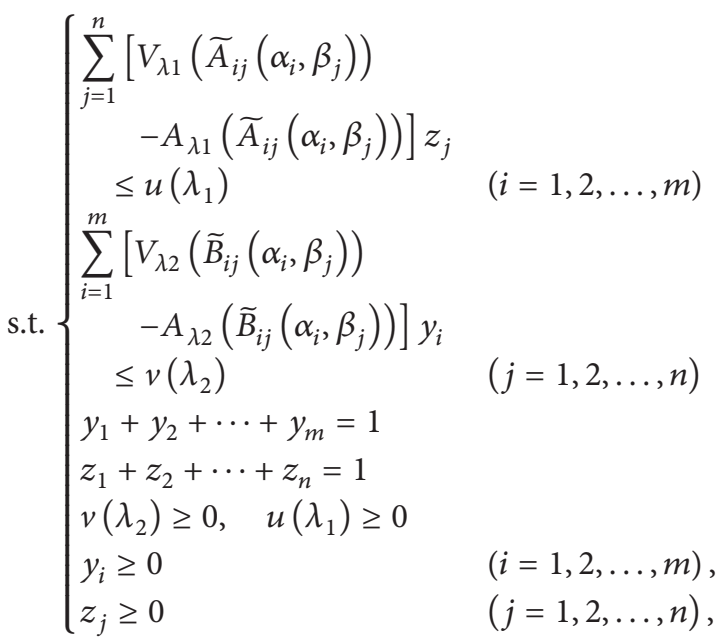

where $y_{i}(i=1,2, \ldots, m), z_{j}(j=1,2, \ldots, n), u\left(\lambda_{1}\right)$, and $v\left(\lambda_{2}\right)$ are decision variables.

According to Theorem 11, if $\left(\mathbf{y}^{*}, \mathbf{z}^{*}, u^{*}\left(\lambda_{1}\right), v^{*}\left(\lambda_{2}\right)\right)$ is a solution of the above parametric bilinear programming model (i.e., (22)), then $u^{*}\left(\lambda_{1}\right)=\mathbf{y}^{* T} \widetilde{\mathbf{A}}_{\lambda_{1}} \mathbf{z}^{*}=$ $\sum_{j=1}^{n} \sum_{i=1}^{m}\left[V_{\lambda 1}\left(\widetilde{A}_{i j}\left(\alpha_{i}, \beta_{j}\right)\right)-A_{\lambda 1}\left(\widetilde{A}_{i j}\left(\alpha_{i}, \beta_{j}\right)\right)\right] y_{i}^{*} z_{j}^{*}, v^{*}\left(\lambda_{2}\right)=$ $\mathbf{y}^{* T} \widetilde{\mathbf{B}}_{\lambda_{2}} \mathbf{z}^{*}=\sum_{j=1}^{n} \sum_{i=1}^{m}\left[V_{\lambda 2}\left(\widetilde{B}_{i j}\left(\alpha_{i}, \beta_{j}\right)\right)-A_{\lambda 2}\left(\widetilde{B}_{i j}\left(\alpha_{i}, \beta_{j}\right)\right)\right]$ $y_{i}^{*} z_{j}^{*}$, and $\mathbf{y}^{* T}\left(D_{\lambda_{1}}(\widetilde{\mathbf{A}})+D_{\lambda_{2}}(\widetilde{\mathbf{B}})\right) \mathbf{z}^{*}-u^{*}\left(\lambda_{1}\right)-v^{*}\left(\lambda_{2}\right)=0$.

Notice that $y_{i}^{*} \geq 0, z_{j}^{*} \geq 0$, and $V_{\lambda}(\widetilde{a})$ and $A_{\lambda}(\widetilde{a})$ are, respectively, continuous nondecreasing and nonincreasing functions of the parameter $\lambda \in[0,1]$ if $\tilde{a}$ is a nonnegative TrIFN. Then, $u^{*}\left(\lambda_{1}\right)$ and $v^{*}\left(\lambda_{2}\right)$ are monotonic and nondecreasing functions of the parameters $\lambda_{1} \in[0,1]$ and $\lambda_{2} \epsilon$ $[0,1]$, respectively. Thus, the satisfying Nash equilibrium values of players I and II are obtained as $\left[u^{*}(0), u^{*}(1)\right]$ and $\left[v^{*}(0), v^{*}(1)\right]$, respectively, and can be written as the TrIFNs $\left\{\left\langle\left(\bar{y}^{*}, \bar{z}^{*}\right), u^{*}(0), 1-u^{*}(1)\right\rangle\right\}$ and $\left\{\left\langle\left(\bar{y}^{*}, \bar{z}^{*}\right), v^{*}(0), 1-\right.\right.$ $\left.\left.v^{*}(1)\right\rangle\right\}$, where $\left(\bar{y}^{*}, \bar{z}^{*}\right)$ represents a mixed situation. Thus, 
the $\widetilde{u}^{*}\left(\bar{y}^{*}, \bar{z}^{*}\right)$ and $\widetilde{v}^{*}\left(\bar{y}^{*}, \bar{z}^{*}\right)$ are Nash equilibrium values of players I and II, respectively.

In particular, for the parameters $\lambda_{1}=0$ and $\lambda_{2}=0,(22)$ becomes the bilinear programming model as follows:

$$
\begin{aligned}
& \max \left\{\sum _ { j = 1 } ^ { n } \sum _ { i = 1 } ^ { m } y _ { i } \left[V_{\mu}\left(\widetilde{A}_{i j}\left(\alpha_{i}, \beta_{j}\right)\right)-A_{v}\left(\widetilde{A}_{i j}\left(\alpha_{i}, \beta_{j}\right)\right)\right.\right. \\
& \left.+V_{\mu}\left(\widetilde{B}_{i j}\left(\alpha_{i}, \beta_{j}\right)\right)-A_{v}\left(\widetilde{B}_{i j}\left(\alpha_{i}, \beta_{j}\right)\right)\right] z_{j} \\
& -u(0)-v(0)\}
\end{aligned}
$$$$
\text { s.t. }\left\{\begin{array}{l}
\sum_{j=1}^{n}\left[V_{\mu}\left(\widetilde{A}_{i j}\left(\alpha_{i}, \beta_{j}\right)\right)\right. \\
\left.\quad-A_{v}\left(\widetilde{A}_{i j}\left(\alpha_{i}, \beta_{j}\right)\right)\right] z_{j} \\
\leq u(0) \\
\left.\quad-A_{v}\left(\widetilde{B}_{i j}\left(\alpha_{i}, \beta_{j}\right)\right)\right] y_{i} \\
\quad \leq \quad v(0) \\
\sum_{i=1}^{m}\left[V_{\mu}\left(\widetilde{B}_{i j}\left(\alpha_{i}, \beta_{j}\right)\right)\right. \\
z_{1}+z_{2}+\cdots+z_{n}=1 \\
z_{j} \geq 0, \quad y_{i} \geq 0 \\
u(0) \geq 0, \quad v(0) \geq 0,
\end{array} \quad(i=1,2, \ldots, n)\right.
$$

where $u(0)=V_{\mu}(\tilde{u}(\bar{y}, \bar{z}))-A_{v}(\tilde{u}(\bar{y}, \bar{z})), v(0)=V_{\mu}(\widetilde{v}(\bar{y}, \bar{z}))-$ $A_{v}(\widetilde{v}(\bar{y}, \bar{z})), y_{i}(i=1,2, \ldots, m), z_{j}(j=1,2, \ldots, n)$, $u(0)$, and $v(0)$ are decision variables. The solution of (23) can be obtained by Lemke-Howson's algorithm (Lemke and Howson [24]) and denoted by $\left(\mathbf{y}^{* T}, \mathbf{z}^{* T}, V_{\mu}\left(\tilde{u}^{*}\left(\bar{y}^{*}, \bar{z}^{*}\right)\right)-\right.$ $\left.A_{v}\left(\widetilde{u}^{*}\left(\bar{y}^{*}, \bar{z}^{*}\right)\right), V_{\mu}\left(\widetilde{v}^{*}\left(\bar{y}^{*}, \bar{z}^{*}\right)\right)-A_{v}\left(\widetilde{v}^{*}\left(\bar{y}^{*}, \bar{z}^{*}\right)\right)\right)$.

Similarly, for the parameters $\lambda_{1}=1$ and $\lambda_{2}=1$, (22) becomes the bilinear programming model as follows:

$$
\begin{aligned}
& \max \left\{\sum _ { j = 1 } ^ { n } \sum _ { i = 1 } ^ { m } y _ { i } \left[V_{v}\left(\widetilde{A}_{i j}\left(\alpha_{i}, \beta_{j}\right)\right)-A_{\mu}\left(\widetilde{A}_{i j}\left(\alpha_{i}, \beta_{j}\right)\right)\right.\right. \\
& \left.+V_{v}\left(\widetilde{B}_{i j}\left(\alpha_{i}, \beta_{j}\right)\right)-A_{\mu}\left(\widetilde{B}_{i j}\left(\alpha_{i}, \beta_{j}\right)\right)\right] z_{j} \\
& -u(1)-v(1)\}
\end{aligned}
$$

$$
\text { s.t. }\left\{\begin{array}{l}
\sum_{j=1}^{n}\left[V_{\mu}\left(\widetilde{A}_{i j}\left(\alpha_{i}, \beta_{j}\right)\right)\right. \\
\left.\quad-A_{v}\left(\widetilde{A}_{i j}\left(\alpha_{i}, \beta_{j}\right)\right)\right] z_{j} \\
\leq u(1) \\
\left.\quad-A_{v}\left(\widetilde{B}_{i j}\left(\alpha_{i}, \beta_{j}\right)\right)\right] y_{i} \\
\quad \leq \quad(0) \\
\sum_{1}+y_{2}+\cdots+y_{m}=1 \\
z_{1}+z_{2}+\cdots+z_{n}=1 \\
z_{j} \geq 0, \quad y_{i} \geq 0 \\
u(1) \geq 0, \quad v(1) \geq 0,
\end{array} \quad(j=1,2, \ldots, n)\right.
$$

where $u(1)=V_{v}\left(\widetilde{u}^{*}\left(\bar{y}^{*}, \bar{z}^{*}\right)\right)-A_{\mu}\left(\widetilde{u}^{*}\left(\bar{y}^{*}, \bar{z}^{*}\right)\right), v(1)=$ $V_{v}\left(\widetilde{v}^{*}\left(\bar{y}^{*}, \bar{z}^{*}\right)\right)-A_{\mu}\left(\widetilde{v}^{*}\left(\bar{y}^{*}, \bar{z}^{*}\right)\right), y_{i}(i=1,2, \ldots, m), z_{j}(j=$ $1,2, \ldots, n), u(1)$, and $v(1)$ are decision variables. Likewise, the solution of (24) can be obtained by Lemke-Howson's algorithm (Lemke and Howson [24]) and denoted by $\left(\mathbf{y}^{\prime * T}, \mathbf{z}^{\prime * T}, V_{v}\left(\tilde{u}^{*}\left(\bar{y}^{*}, \bar{z}^{*}\right)\right)-A_{\mu}\left(\tilde{u}^{*}\left(\bar{y}^{*}, \bar{z}^{*}\right)\right), V_{v}\left(\widetilde{v}^{*}\left(\bar{y}^{*}, \bar{z}^{*}\right)\right)-\right.$ $\left.A_{\mu}\left(\widetilde{v}^{*}\left(\bar{y}^{*}, \bar{z}^{*}\right)\right)\right)$.

Thus, we can explicitly obtain the satisfying Nash equilibrium values and corresponding satisfying Nash equilibrium strategies of players I and II through solving the derived two bilinear programming models (i.e., (23) and (24)). Furthermore, according to (22), any satisfying Nash equilibrium values and corresponding satisfying Nash equilibrium strategies of players I and II can be obtained through choosing different parameters $\lambda_{1} \in[0,1]$ and $\lambda_{2} \in$ $[0,1]$.

\section{An Example of a Commerce Retailers' Strategy Choice Problem}

Let us consider the case of two commerce retailers $R_{1}$ and $R_{2}$ (i.e., players I and II) making a decision aiming to enhance the satisfaction degrees of customers. As players' judgments for the satisfaction degrees of customers including preference and experience are often vague and players estimate them with their intuitions. And assume that commerce retailers $R_{1}$ and $R_{2}$ are rational; that is, they will choose optimal strategies to maximize their own profits without cooperation. Suppose that retailer $R_{1}$ has two pure strategies: establishing a scientific and rational service system $\alpha_{1}$ and providing customers with satisfaction products $\alpha_{2}$. Retailer $R_{2}$ possesses the same pure strategies as retailer $R_{1}$; that is, the options of retailer $R_{2}$ are establishing a scientific and rational service system $\beta_{1}$ and providing customers with satisfaction products $\beta_{2}$.

Let us consider the following specific TrIFN bimatrix game for this scenario, where the payoff matrices of commerce retailers $R_{1}$ and $R_{2}$ are expressed with TrIFNs as follows: 
TABLE 1: Satisfying Nash equilibrium values and corresponding strategies of commerce retailers.

\begin{tabular}{lccccc}
\hline & Parameters & & $R_{1}$ & & $R_{2}$ \\
$\lambda_{1}$ & $\lambda_{2}$ & $\mathbf{y}^{* T}$ & $u^{*}\left(\lambda_{1}\right)$ & $(0.311,0.689)$ & $v^{*}\left(\lambda_{2}\right)$ \\
\hline 0 & 0 & $(0.484,0.516)$ & 7.94 & $(0.311,0.689)$ \\
0 & 0.3 & $(0.492,0.508)$ & 7.94 & $(0.311,0.689)$ & 11.98 \\
0 & 0.5 & $(0.498,0.502)$ & 7.94 & $(0.311,0.689)$ \\
0 & 0.8 & $(0.495,0.505)$ & 7.94 & $(0.311,0.689)$ & 12.98 \\
0 & 1 & $(0.490,0.510)$ & 7.94 & 14.48 \\
\hline
\end{tabular}

TABLE 2: Satisfying Nash equilibrium values and corresponding strategies of commerce retailers.

\begin{tabular}{cccccc}
\hline & Parameters & & $R_{1}$ & & $R_{2}$ \\
$\lambda_{1}$ & $\lambda_{2}$ & $\mathbf{y}^{* T}$ & $u^{*}\left(\lambda_{1}\right)$ & $\mathbf{z}^{* T}\left(\lambda_{2}\right)$ \\
\hline 0.5 & 0 & $(0.484,0.516)$ & 11.19 & $(0.266,0.734)$ \\
0.5 & 0.3 & $(0.492,0.508)$ & 11.19 & $(0.266,0.734)$ & 10.46 \\
0.5 & 0.5 & $(0.498,0.502)$ & 11.19 & $(0.266,0.734)$ & 11.98 \\
0.5 & 0.8 & $(0.495,0.505)$ & 11.19 & $(0.266,0.734)$ & 12.98 \\
0.5 & 1 & $(0.490,0.510)$ & 11.19 & $(0.266,0.734)$ & 14.48 \\
\hline
\end{tabular}

$$
\begin{aligned}
& \widetilde{\mathbf{A}}=\left(\begin{array}{lll}
\left\{\left\langle\left(\alpha_{1}, \beta_{1}\right),(50,60,70,80), 0.8,0.1\right\rangle\right\} & \left\{\left\langle\left(\alpha_{1}, \beta_{2}\right),(30,40,50,60), 0.4,0.3\right\rangle\right\} \\
\left\{\left\langle\left(\alpha_{2}, \beta_{1}\right),(20,30,40,50), 0.5,0.4\right\rangle\right\} & \left\{\left\langle\left(\alpha_{2}, \beta_{2}\right),(40,50,60,70), 0.6,0.2\right\rangle\right\}
\end{array}\right), \\
& \widetilde{\mathbf{B}}=\left(\begin{array}{ll}
\left\{\left\langle\left(\alpha_{1}, \beta_{1}\right),(40,50,60,70), 0.7,0.1\right\rangle\right\} & \left\{\left\langle\left(\alpha_{1}, \beta_{2}\right),(30,40,50,60), 0.7,0.2\right\rangle\right\} \\
\left\{\left\langle\left(\alpha_{2}, \beta_{1}\right),(20,30,40,50), 0.5,0.3\right\rangle\right\} & \left\{\left\langle\left(\alpha_{2}, \beta_{2}\right),(50,60,70,80), 0.8,0.1\right\rangle\right\}
\end{array}\right),
\end{aligned}
$$

where the $\operatorname{TrIFN}\left\{\left\langle\left(\alpha_{1}, \beta_{1}\right),(50,60,70,80), 0.8,0.1\right\rangle\right\}$ in the payoff matrix $\widetilde{\mathbf{A}}$ means that the payoff is between 50 and 80 , and satisfaction (or membership) degree of customers is 0.8 , and the dissatisfaction (or nonmembership) degree of customers is 0.1 for commerce retailer $R_{1}$ if he/she adopts the pure strategy $\alpha_{1}$ and commerce retailer $R_{2}$ also adopts the pure strategy $\beta_{1}$. Other entries in the TrIFN payoff matrices $\widetilde{\mathbf{A}}$ and $\widetilde{\mathbf{B}}$ can be similarly explained.

According to (16) and (17), respectively, the value indexes and the ambiguity-indexes of $\left.\widetilde{A}\left(\alpha_{i}, \beta_{j}\right)\right), i=1,2, j=1,2$, can be obtained as follows:

$$
\begin{gathered}
V_{\lambda 1}\left(\widetilde{A}\left(\alpha_{1}, \beta_{1}\right)\right)=26+3.25 \lambda_{1}, \\
A_{\lambda 1}\left(\widetilde{A}\left(\alpha_{1}, \beta_{1}\right)\right)=7.5-0.83 \lambda_{1}, \\
V_{\lambda 1}\left(\widetilde{A}\left(\alpha_{1}, \beta_{2}\right)\right)=9+6.75 \lambda_{1}, \\
A_{\lambda 1}\left(\widetilde{A}\left(\alpha_{1}, \beta_{2}\right)\right)=5.83-2.5 \lambda_{1}, \\
V_{\lambda 1}\left(\widetilde{A}\left(\alpha_{2}, \beta_{1}\right)\right)=8.75+1.75 \lambda_{1}, \\
A_{\lambda 1}\left(\widetilde{A}\left(\alpha_{2}, \beta_{1}\right)\right)=5-0.83 \lambda_{1},
\end{gathered}
$$

$$
\begin{gathered}
V_{\lambda 1}\left(\widetilde{A}\left(\alpha_{2}, \beta_{2}\right)\right)=16+5.5 \lambda_{1}, \\
A_{\lambda 1}\left(\widetilde{A}\left(\alpha_{2}, \beta_{2}\right)\right)=6.67-1.67 \lambda_{1} .
\end{gathered}
$$

According to (18), the differences of the value indexes to the ambiguity indexes for the $\left.\operatorname{TrIFNs} \widetilde{A}\left(\alpha_{i}, \beta_{j}\right)\right), i=1,2, j=$ 1,2 , can be obtained as follows:

$$
\begin{aligned}
D_{\lambda 1}\left(\widetilde{A}\left(\alpha_{1}, \beta_{1}\right)\right) & =V_{\lambda 1}\left(\widetilde{A}\left(\alpha_{1}, \beta_{1}\right)\right)-A_{\lambda 1}\left(\widetilde{A}\left(\alpha_{1}, \beta_{1}\right)\right) \\
& =18.5+4.08 \lambda_{1}, \\
D_{\lambda 1}\left(\widetilde{A}\left(\alpha_{1}, \beta_{2}\right)\right) & =V_{\lambda 1}\left(\widetilde{A}\left(\alpha_{1}, \beta_{2}\right)\right)-A_{\lambda 1}\left(\widetilde{A}\left(\alpha_{1}, \beta_{2}\right)\right) \\
& =3.17+9.25 \lambda_{1}, \\
D_{\lambda 1}\left(\widetilde{A}\left(\alpha_{2}, \beta_{1}\right)\right) & =V_{\lambda 1}\left(\widetilde{A}\left(\alpha_{2}, \beta_{1}\right)\right)-A_{\lambda 1}\left(\widetilde{A}\left(\alpha_{2}, \beta_{1}\right)\right) \\
& =3.75+2.58 \lambda_{1}, \\
D_{\lambda 1}\left(\widetilde{A}\left(\alpha_{2}, \beta_{2}\right)\right) & =V_{\lambda 1}\left(\widetilde{A}\left(\alpha_{2}, \beta_{2}\right)\right)-A_{\lambda 1}\left(\widetilde{A}\left(\alpha_{2}, \beta_{2}\right)\right) \\
& =9.83+7.17 \lambda_{1} .
\end{aligned}
$$


TABLE 3: Satisfying Nash equilibrium values and corresponding strategies of commerce retailers.

\begin{tabular}{lccccc}
\hline & Parameters & & $R_{1}$ & & $R_{2}$ \\
$\lambda_{1}$ & $\lambda_{2}$ & $\mathbf{y}^{* T}$ & $u^{*}\left(\lambda_{1}\right)$ & $(0.220,0.780)$ & $v^{*}\left(\lambda_{2}\right)$ \\
\hline 1 & 0 & $(0.484,0.516)$ & 14.65 & $(0.220,0.780)$ & 10.46 \\
1 & 0.3 & $(0.492,0.508)$ & 14.65 & $(0.220,0.780)$ & 11.98 \\
1 & 0.5 & $(0.498,0.502)$ & 14.65 & $(0.220,0.780)$ & 12.98 \\
1 & 0.8 & $(0.495,0.505)$ & 14.65 & $(0.220,0.780)$ & 14.48 \\
1 & 1 & $(0.490,0.510)$ & 14.65 & & 15.47 \\
\hline
\end{tabular}

TABLE 4: Satisfying Nash equilibrium values and corresponding strategies of commerce retailers.

\begin{tabular}{lccccc}
\hline & Parameters & & $R_{1}$ & & $R_{2}$ \\
$\lambda_{1}$ & $\lambda_{2}$ & $\mathbf{y}^{* T}$ & $u^{*}\left(\lambda_{1}\right)$ & $\mathbf{z}^{* T}\left(\lambda_{2}\right)$ \\
\hline 0 & 0 & $(0.516,0.484)$ & 7.94 & $(0.311,0.689)$ \\
0.3 & 0 & $(0.516,0.484)$ & 9.86 & $(0.284,0.716)$ \\
0.5 & 0 & $(0.516,0.484)$ & 11.19 & $(0.266,0.734)$ & 10.46 \\
0.8 & 0 & $(0.516,0.484)$ & 13.24 & $(0.239,0.761)$ & 10.46 \\
1 & 0 & $(0.516,0.484)$ & 14.65 & $(0.220,0.780)$ & 10.46 \\
\hline
\end{tabular}

In the same way, we can obtain that the differences of the value indexes to the ambiguity indexes for the TrIFNs $\left.\widetilde{B}\left(\alpha_{i}, \beta_{j}\right)\right)(i=1,2, j=1,2)$ can be obtained as follows:

$$
\begin{aligned}
D_{\lambda 2}\left(\widetilde{B}\left(\alpha_{1}, \beta_{1}\right)\right) & =V_{\lambda 2}\left(\widetilde{B}\left(\alpha_{1}, \beta_{1}\right)\right)-A_{\lambda 2}\left(\widetilde{B}\left(\alpha_{1}, \beta_{1}\right)\right) \\
& =11.75+7.17 \lambda_{2}, \\
D_{\lambda 2}\left(\widetilde{B}\left(\alpha_{1}, \beta_{2}\right)\right) & =V_{\lambda 2}\left(\widetilde{B}\left(\alpha_{1}, \beta_{2}\right)\right)-A_{\lambda 2}\left(\widetilde{B}\left(\alpha_{1}, \beta_{2}\right)\right) \\
& =9.08+3.08 \lambda_{2}, \\
D_{\lambda 2}\left(\widetilde{B}\left(\alpha_{2}, \beta_{1}\right)\right) & =V_{\lambda 2}\left(\widetilde{B}\left(\alpha_{2}, \beta_{1}\right)\right)-A_{\lambda 2}\left(\widetilde{B}\left(\alpha_{2}, \beta_{1}\right)\right) \\
& =2.92+5.17 \lambda_{2}, \\
D_{\lambda 2}\left(\widetilde{B}\left(\alpha_{2}, \beta_{2}\right)\right) & =V_{\lambda 2}\left(\widetilde{B}\left(\alpha_{2}, \beta_{2}\right)\right)-A_{\lambda 2}\left(\widetilde{B}\left(\alpha_{2}, \beta_{2}\right)\right) \\
& =18.5+4.08 \lambda_{2} .
\end{aligned}
$$

Using (22), the parametric bilinear programming model is constructed as follows:

$$
\begin{aligned}
\max & \left\{\left(30.25+4.08 \lambda_{1}+7.17 \lambda_{2}\right) y_{1} z_{1}\right. \\
& +\left(12.25+9.25 \lambda_{1}+3.08 \lambda_{2}\right) y_{1} z_{2} \\
& +\left(6.67+2.58 \lambda_{1}+5.17 \lambda_{2}\right) y_{2} z_{1} \\
& \left.+\left(28.33+7.17 \lambda_{1}+4.08 \lambda_{2}\right) y_{2} z_{2}-u\left(\lambda_{1}\right)-v\left(\lambda_{2}\right)\right\}
\end{aligned}
$$

$$
\text { s.t. }\left\{\begin{array}{l}
\left(18.5+4.08 \lambda_{1}\right) z_{1}+\left(3.17+9.25 \lambda_{1}\right) z_{2} \leq u\left(\lambda_{1}\right) \\
\left(3.75+2.58 \lambda_{1}\right) z_{1}+\left(9.83+7.17 \lambda_{1}\right) z_{2} \leq u\left(\lambda_{1}\right) \\
\left(11.75+7.17 \lambda_{2}\right) y_{1}+\left(9.08+3.08 \lambda_{2}\right) y_{2} \leq v\left(\lambda_{2}\right) \\
\left(2.92+5.17 \lambda_{2}\right) y_{1}+\left(18.5+4.08 \lambda_{2}\right) y_{2} \leq v\left(\lambda_{2}\right) \\
y_{1}+y_{2}=1 \\
z_{1}+z_{2}=1 \quad \\
u\left(\lambda_{1}\right) \geq 0, \quad v\left(\lambda_{2}\right) \geq 0 \\
y_{i} \geq 0 \quad(i=1,2), \\
z_{j} \geq 0 \quad(j=1,2) .
\end{array}\right.
$$

It is easily seen that, for the parameters $\lambda_{1} \in[0,1]$ and $\lambda_{2} \in[0,1]$, solving (29), we can obtain the satisfying Nash equilibrium values and corresponding satisfying Nash equilibrium strategies of commerce retailers $R_{1}$ and $R_{2}$, respectively, depicted as in Tables 1, 2, 3, 4, 5, and 6 .

It can be easily seen from Tables 1-3 (or Tables 4-6) that the satisfying Nash equilibrium value of a player (i.e., I/commerce retailer $R_{1}$ or II/ $R_{2}$ ) only depends on his/her own preference/parameter regardless of other player's preferences/parameters. However, strategy choice of a player is only affected by other player's preferences/parameters.

\section{Conclusion}

In some situations, determining payoffs of bimatrix games absolutely depends on players' judgments and intuition, which are often vague and not easy to be represented with crisp values and fuzzy numbers. In the above, we model TrIFN bimatrix games and develop the parametric bilinear programming models and method by using the new order relation of TrIFNs given in this paper. The developed models and method can simplify the calculation of Nash equilibrium solutions of TrIFN bimatrix games. 
TABLE 5: Satisfying Nash equilibrium values and corresponding strategies of commerce retailers.

\begin{tabular}{lccccc}
\hline & Parameters & & $R_{1}$ & & \\
$\lambda_{1}$ & $\lambda_{2}$ & $\mathbf{y}^{* T}$ & $u^{*}\left(\lambda_{1}\right)$ & $\mathbf{z}^{* T}$ & $v^{*}\left(\lambda_{2}\right)$ \\
\hline 0 & 0.5 & $(0.502,0.498)$ & 7.94 & $(0.311,0.689)$ & 12.99 \\
0.3 & 0.5 & $(0.502,0.498)$ & 9.86 & $(0.284,0.716)$ & 12.99 \\
0.5 & 0.5 & $(0.502,0.498)$ & 11.19 & $(0.266,0.734)$ & 12.99 \\
0.8 & 0.5 & $(0.502,0.498)$ & 13.24 & $(0.239,0.761)$ & 12.99 \\
1 & 0.5 & $(0.502,0.498)$ & 14.65 & $(0.220,0.780)$ & 12.99 \\
\hline
\end{tabular}

TABLE 6: Satisfying Nash equilibrium values and corresponding strategies of commerce retailers.

\begin{tabular}{lccccc}
\hline & Parameters & & $R_{1}$ & & $R_{2}$ \\
$\lambda_{1}$ & $\lambda_{2}$ & $\mathbf{y}^{* T}$ & $u^{*}\left(\lambda_{1}\right)$ & $\mathbf{z}^{* T}$ & $v^{*}\left(\lambda_{2}\right)$ \\
\hline 0 & 1 & $(0.490,0.510)$ & 7.94 & $(0.311,0.689)$ & 15.47 \\
0.3 & 1 & $(0.490,0.510)$ & 9.86 & $(0.284,0.716)$ & 15.47 \\
0.5 & 1 & $(0.490,0.510)$ & 11.19 & $(0.266,0.734)$ & 15.47 \\
0.8 & 1 & $(0.490,0.510)$ & 13.24 & $(0.239,0.761)$ & 15.47 \\
1 & 1 & $(0.490,0.510)$ & 14.65 & $(0.220,0.780)$ & 15.47 \\
\hline
\end{tabular}

Furthermore, it is easy to see that the models and method proposed in this paper may be extended to TrIFN multiobjective bimatrix games. And more effective methods of TrIFN bimatrix games will be investigated in the near future. Also the proposed models and method may be applied to solving many competitive decision problems in similar fields such as management, supply chain, and advertising, although they are illustrated with the example of the commerce retailers' strategy choice problem in this paper.

\section{Acknowledgments}

The authors would like to thank the valuable comments and also appreciate the constructive suggestions from the anonymous referees. This research was supported by the Key Program of National Natural Science Foundation of China (no. 71231003), the National Natural Science Foundation of China (nos. 71171055, 71101033, and 71001015), the Program for New Century Excellent Talents in University (the Ministry of Education of China, NCET-10-0020), and the Specialized Research Fund for the Doctoral Program of Higher Education of China (no. 20113514110009).

\section{References}

[1] L. A. Zadeh, "Fuzzy sets," Information and Control, vol. 8, no. 3, pp. 338-353, 1965.

[2] V. Vidyottama, S. Chandra, and C. R. Bector, "Bi-matrix games with fuzzy goals and fuzzy pay-offs," Fuzzy Optimization and Decision Making, vol. 3, no. 4, pp. 327-344, 2004.

[3] T. Maeda, "Characterization of the equilibrium strategy of the bimatrix game with fuzzy payoff," Journal of Mathematical Analysis and Applications, vol. 251, no. 2, pp. 885-896, 2000.

[4] C. R. Bector and S. Chandra, Fuzzy Mathematical Programming and Fuzzy Matrix Games, vol. 169 of Studies in Fuzziness and Soft Computing, Springer, Berlin, Germany, 2005.
[5] M. Larbani, "Solving bimatrix games with fuzzy payoffs by introducing nature as a third player," Fuzzy Sets and Systems, vol. 160, no. 5, pp. 657-666, 2009.

[6] K. T. Atanassov, "Intuitionistic fuzzy sets," Fuzzy Sets and Systems, vol. 20, no. 1, pp. 87-96, 1986.

[7] G. Wei, "Some arithmetic aggregation operators with intuitionistic trapezoidal fuzzy numbers and their application to group decision making," Journal of Computers, vol. 5, no. 3, pp. 345351, 2010.

[8] J. Ye, "Multicriteria group decision-making method using vector similarity measures for trapezoidal intuitionistic fuzzy numbers," Group Decision and Negotiation, vol. 21, no. 4, pp. 519-530, 2010.

[9] S.-p. Wan, "Power average operators of trapezoidal intuitionistic fuzzy numbers and application to multi-attribute group decision making," Applied Mathematical Modelling, vol. 37, no. 6, pp. 4112-4126, 2013.

[10] Z. Zhang, D. Tian, and K. Li, "Parameterized intuitionistic fuzzy trapezoidal operators and their application to multiple attribute groupdecision making," Journal of Intelligent and Fuzzy Systems. In press.

[11] B. Asady and A. Zendehnam, "Ranking fuzzy numbers by distance minimization," Applied Mathematical Modelling, vol. 31, no. 11, pp. 2589-2598, 2007.

[12] P. Xu, X. Su, J. Wu, X. Sun, Y. Zhang, and Y. Deng, "A note on ranking generalized fuzzy numbers," Expert Systems with Applications, vol. 39, no. 7, pp. 6454-6457, 2012.

[13] V. L. G. Nayagam, G. Venkateshwari, and G. Sivaraman, "Ranking of intuitionistic fuzzy numbers," in Proceedings of the IEEE International Conference on Fuzzy Systems (FUZZ-IEEE '08), pp. 1971-1974, Hong Kong, June 2008.

[14] X. Zhang and Z. Xu, "A new method for ranking intuitionistic fuzzy values and its application in multi-attribute decision making," Fuzzy Optimization and Decision Making, vol. 11, no. 2, pp. 135-146, 2012.

[15] E. Jafarian and M. Ali Rezvani, "A valuation-based method for ranking the intuitionistic fuzzy numbers," Journal of Intelligent \& Fuzzy Systems, vol. 24, no. 1, pp. 133-144, 2013. 
[16] D.-F. Li, "A ratio ranking method of triangular intuitionistic fuzzy numbers and its application to MADM problems," Computers \& Mathematics with Applications, vol. 60, no. 6, pp. 15571570, 2010.

[17] D.-F. Li, J. X. Nan, and M. J. Zhang, "A ranking method of triangular intuitionistic fuzzy numbers and application to decision making," International Journal of Computational Intelligence Systems, vol. 3, no. 5, pp. 522-530, 2010.

[18] J. Ye, "Expected value method for intuitionistic trapezoidal fuzzy multicriteria decision-making problems," Expert Systems with Applications, vol. 38, no. 9, pp. 11730-11734, 2011.

[19] D. Dubois and H. Prade, Fuzzy Sets and Systems: Theory and Applications, vol. 144 of Mathematics in Science and Engineering, Academic Press, New York, NY, USA, 1980.

[20] X. Wang and E. E. Kerre, "Reasonable properties for the ordering of fuzzy quantities (I)," Fuzzy Sets and Systems, vol. 118, no. 3, pp. 375-385, 2001.

[21] G. Owen, Game Theory, Academic Press, New York, NY, USA, 2nd edition, 1982.

[22] J. F. Nash Jr., "Equilibrium points in $n$-person games," Proceedings of the National Academy of Sciences of the United States of America, vol. 36, pp. 48-49, 1950.

[23] O. L. Mangasarian and H. Stone, "Two-person nonzero-sum games and quadratic programming," Journal of Mathematical Analysis and Applications, vol. 9, pp. 348-355, 1964.

[24] C. E. Lemke and J. T. Howson, Jr., "Equilibrium points of bimatrix games," Journal of the Society for Industrial and Applied Mathematics, vol. 12, pp. 413-423, 1964. 


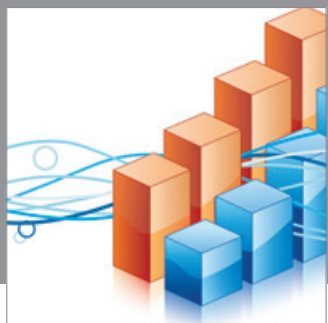

Advances in

Operations Research

mansans

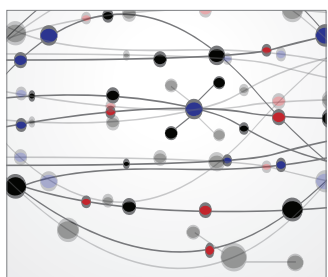

The Scientific World Journal
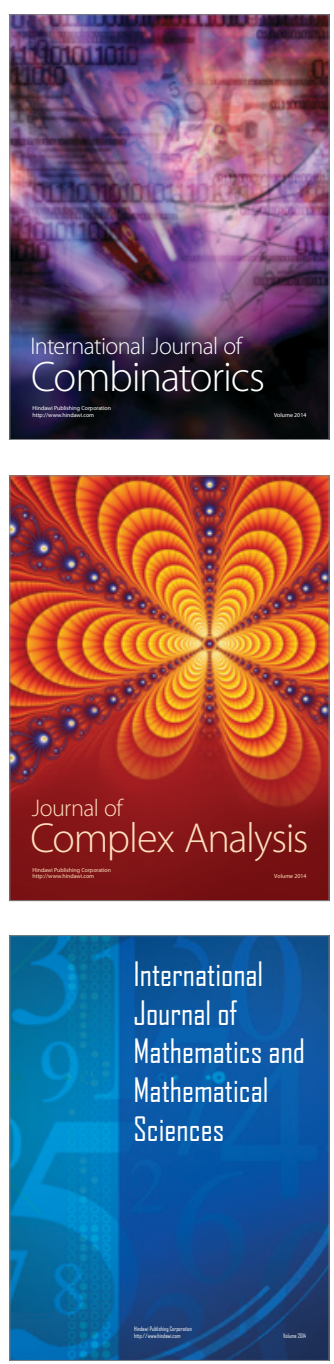
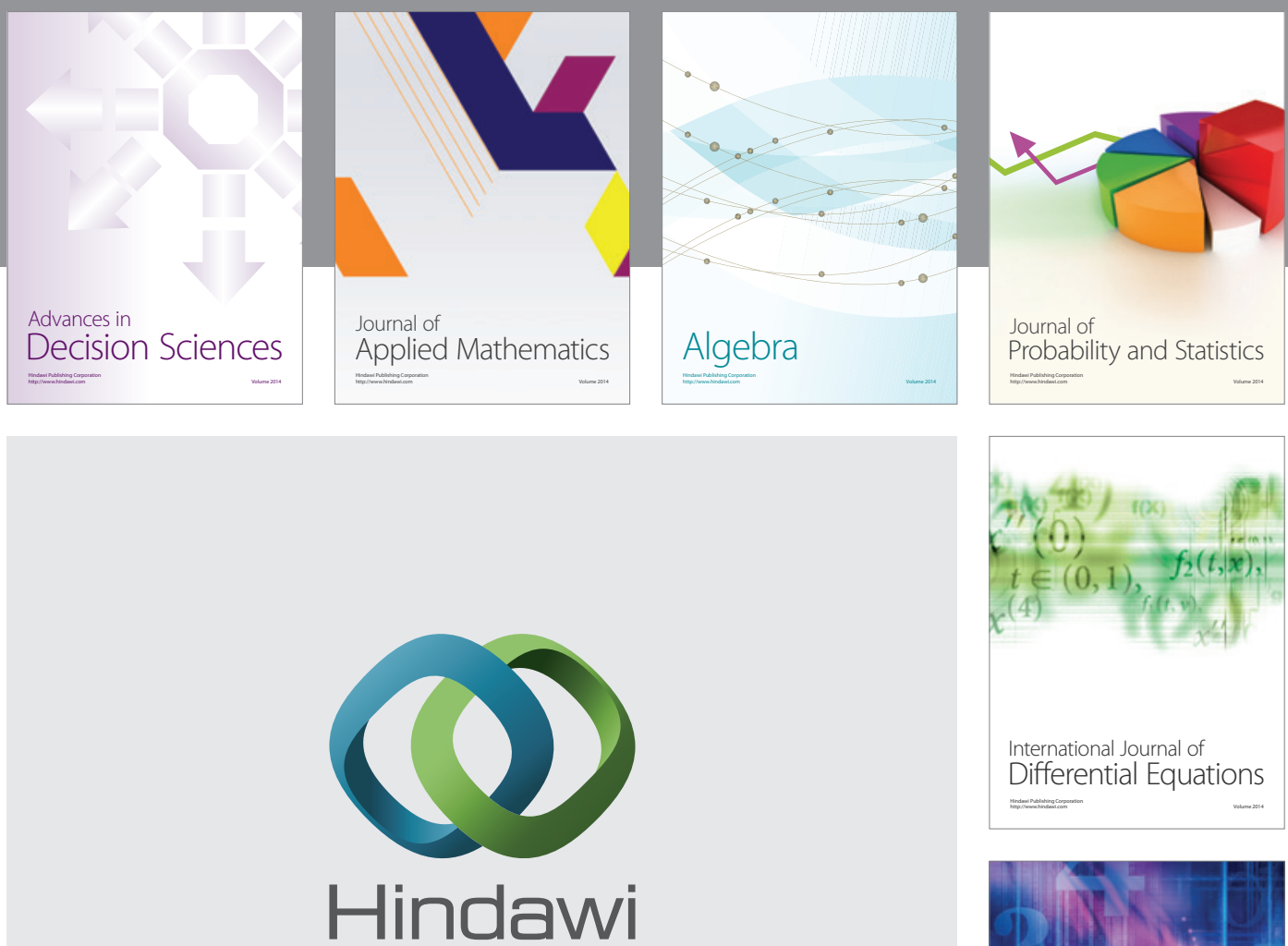

Submit your manuscripts at http://www.hindawi.com
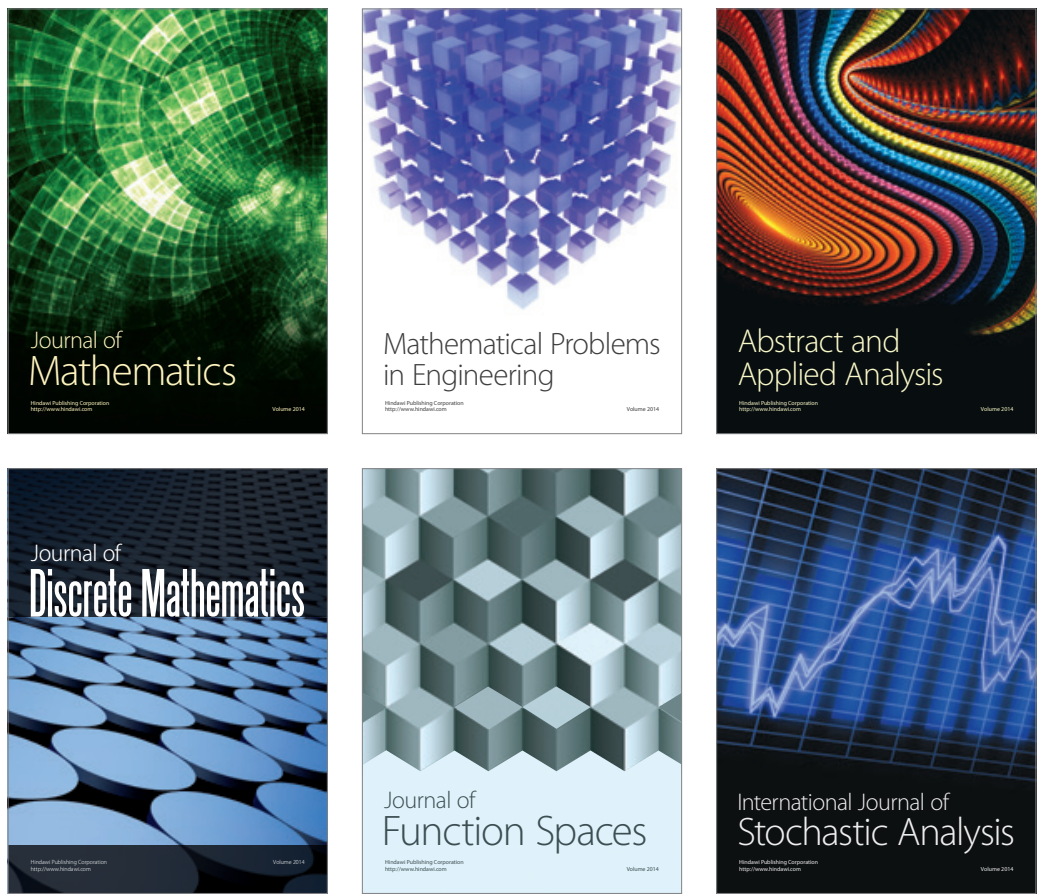

Journal of

Function Spaces

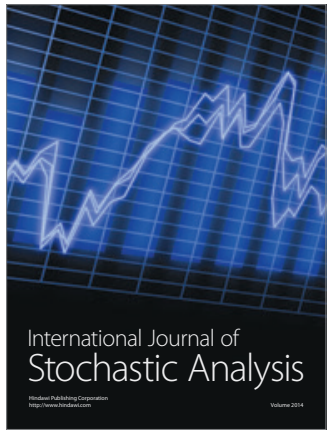

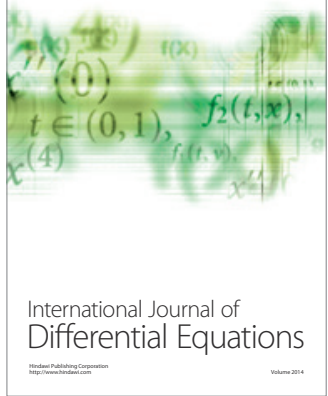
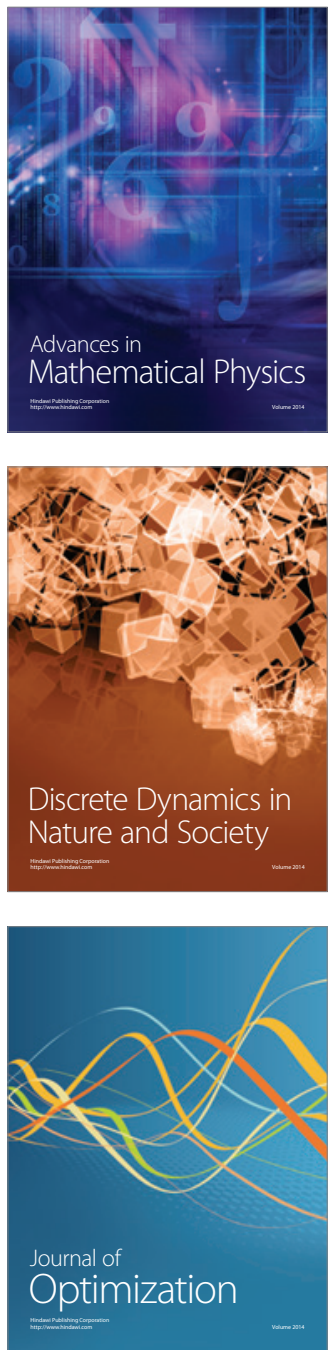